\title{
Enhanced, risk-based system development process: a case study from the Belgian transmission network
}

\author{
Waqquas Bukhsh, Keith Bell \\ Dept. of Electronic and Electrical Engineering, \\ University of Strathclyde, \\ Glasgow, United Kingdom. \\ \{waqquas.bukhsh, keith.bell\}@strath.ac.uk
}

\author{
Arnaud Vergnol, Antoine Weynants, Jonathan Sprooten \\ Elia, Belgian Transmission System Operator, \\ Brussels, Belgium. \\ \{arnaud.vergnol, antoine.weynants, jonathan.sprooten\}@elia.be
}

\begin{abstract}
A large share of renewable generation raises some unique challenges and is forcing change in the operation and planning of electricity transmission systems. In this paper, using real transmission network data representing a part of the Belgian network, the need for building a large set of credible future operating conditions to identify and validate system development decisions is demonstrated. The paper argues that the traditional approach of checking system development options on a few selected operating conditions is no longer adequate in a power system with high penetration of variable generation. An application of a clustering algorithm is demonstrated to build a representative set of operating conditions for system development that not only gives better representation of future operation, but also provides a system development planner with greater confidence with respect to investments that are likely to be necessary. The paper describes the way in which operability of the future system is evaluated and possible need for reinforcement is identified. A discussion is presented of the meeting of demand under initially intact network conditions and while facilitating maintenance outages securely.
\end{abstract}

Index Terms-transmission planning; renewable energy sources; optimal power flow.

\section{INTRODUCTION}

A transmission system operator (TSO) is responsible for building, maintaining and operating an adequate transmission network to ensure safe system operation and security of supply and for facilitating grid access as well as meeting the national and regional objectives. System development is a crucial part of any TSO's activity and concerns making decisions on the development of the network's power transfer capacity while taking into account overall social welfare in both the short and long term in coordination with neighbouring transmission and distribution systems [1].

In Europe, the need for greater market integration and for economically efficient grid connection of different renewable energy sources (RES) is well recognised [2]-[5]. The challenges associated with reliable and secure operation of a power system increase as the electricity generation mix moves away

The research leading to these results has received funding from the European Union Seventh Framework Programme under Grant Agreement No 608540 . from controllable fossil fuel generation to intermittent and partially controllable generation from RES.

The main role of a transmission system development planner is to propose investments in network facilities that are required in order to enable future operation of the system. A system planner needs to postulate a range of credible future operating states and check the operability of the system under each of them. Due to the large number of uncertainties and the complexity associated with checking the operability of each state, common practice in industry to date has been for a system development planner to consider few operating conditions, e.g. a peak demand case with some generating units unavailable.

Elia is the TSO of the Belgian transmission system. It owns the $150-380 \mathrm{kV}$ transmission system in Belgium and also the majority of the $70 \mathrm{kV}$ network. At the time of writing, Belgium is primarily reliant on generation from nuclear power stations to meet its electricity demand. However, the generation mix is changing and a significant amount of RES has been connected to the Belgian grid. The growth of RES is supported by favourable policies e.g. green certificates that ensure minimum revenue for RES generation. Elia, as a system operator, is also obliged to give priority to generation from RES.

The increasing penetration of RES and particularly its location in Belgium may require significant infrastructure investments to ensure reliability of electricity supply and the economic utilisation of the power available from renewables. The geographical location of Belgium in Europe contributes to increased stress on the country's transmission system due to the growth in highly variable electricity flows resulting from the evolution of the European generation fleet, not least a high concentration of wind energy production units in the north of Germany. In the event of favourable weather conditions, Germany exports surplus wind power, in particular through the Netherlands and to and through Belgium. Belgium's northern border is therefore, and especially in winter when energy comes largely from the north, more under pressure.

In this paper, the problem of identifying the need for investment in a part of the Belgian transmission network for the year 2030 is considered. Using appropriate assumptions on the growth of solar, wind and other generation types, a large set of representative operating conditions is built. The set is reduced using an appropriate clustering algorithm. The 
reduced set of operating conditions forms the basis of a new system development process, developed as part of the GARPUR project [6], that checks the operability of each operating condition and identifies critical operating conditions, i.e. those in which operating standards are breached or for which system operational costs are too expensive relative to the likely cost of a reinforcement that would reduce operational costs. Using real transmission network data, the need to build a large set of credible future operating conditions to validate system development decisions is demonstrated. This paper argues that checking system development decisions on a few operating conditions is no longer adequate in a power system with high penetration of RES.

The main contributions of this paper are as follows:

- a new modelling framework that is appropriate for system development studies;

- application of a clustering algorithm to produce representative operating states;

- proposal of guidelines for system development planners to allow them to identify future needs;

- demonstration of a new framework, clustering and guidelines for a realistic system development case.

The paper is organised as follows. In section II, the GARPUR's system development framework is described. A part of Belgian transmission network that is used to demonstrate the system development framework is presented in section III. In section IV, the results of the generation of credible operating states for the test system and their clustering and assessment are presented. Section V highlights some investment options that the methodology has revealed and present results of the assessment of each investment option. In section VI, along with conclusions, a discussion on the main aspects of the system development approach is provided.

\section{GARPUR'S SYSTEM DEVELOPMENT FRAMEWORK}

The GARPUR project [6] is concerned with an improved understanding of risk attributes that impact power system reliability performance in operational and planning timescales. The main steps of the system development framework proposed in GARPUR are presented in the algorithm below. For more technical details of each step of this algorithm, readers are referred to [7].

The first three steps of the system development framework propose to construct a large set of operating states to represent the year around operation of a power system. This is achieved by constructing operational scenarios that specify hourly profiles of demand, power available from renewables and maintenance of assets for the target year. The operational scenarios are used as in input for a tool modelling the operation of the wholesale energy market. The output of this tool identifies the commitment of thermal generators in accordance with prevailing market rules but otherwise unconstrained by the networks capacity. The year long market solution is converted into hourly snapshots, which are then clustered using an appropriate clustering method. The centroids of the clusters are tested for operability using an optimisation model that

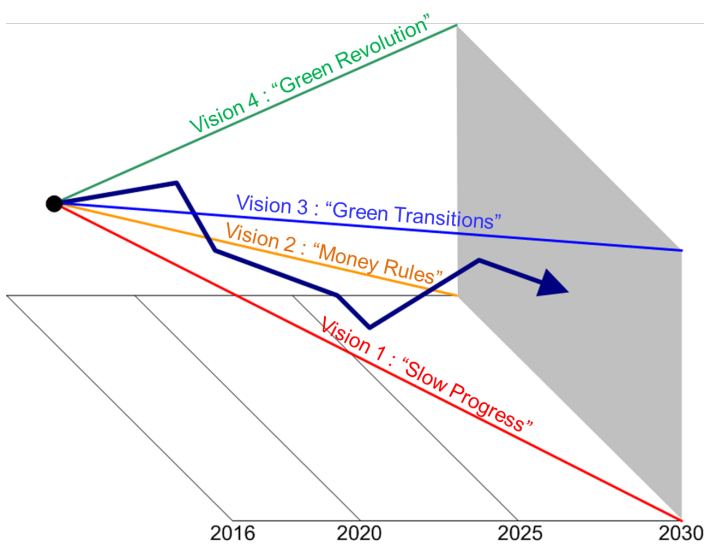

Figure 1. Illustration of ENTSO-e macro-scenarios for the year 2030. Solid blue line with an arrow head represents a possible realisation that is contained by the other four scenarios.

represents the system's behaviour under each scenario, and models both preventive and corrective actions that a system operator may take in real time.

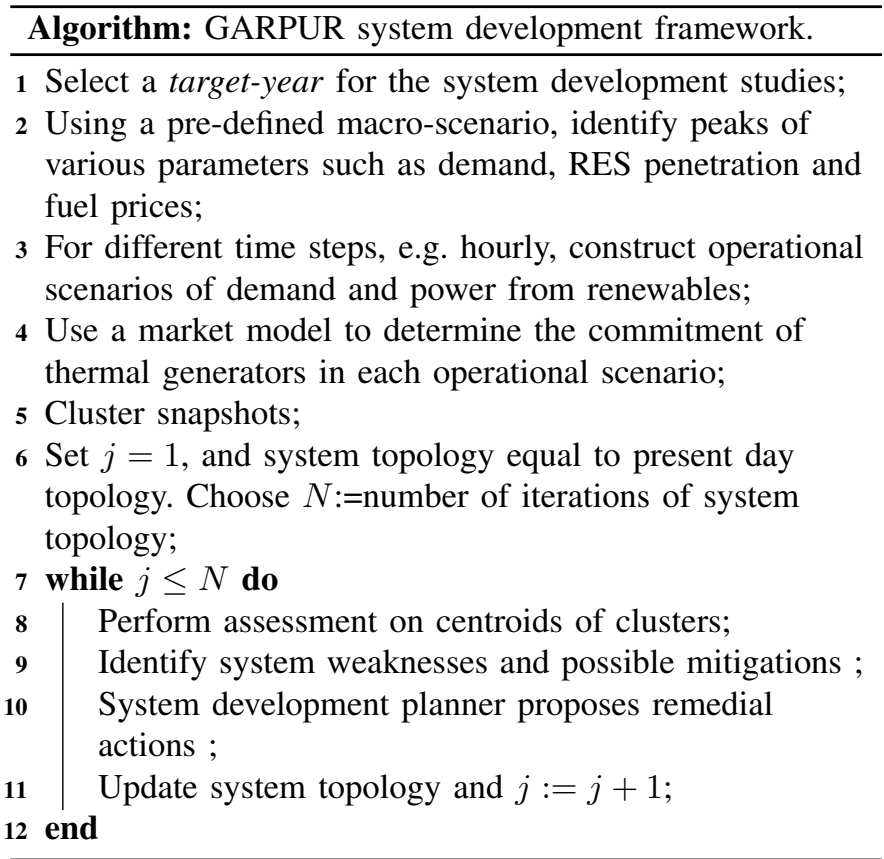

\section{A. Macro-scenarios}

The future operation of a power system depends both on the longer-term market and economic developments that influence the opening and closure of generation capacity and patterns of demand and the ways in which generation and network availability change in the short-term and generation is dispatched to meet demand. All of these factors are uncertain and their collective outcomes are impossible to predict with high confidence. A pragmatic approach is to consider longerterm and shorter-term variations separately. Macro-scenarios are used to model the uncertainty in the long-term evolution of 
parameters that affect future operation of a power system. For example, recent ENTSO-e studies [8] are based on exploration of a time horizon out to 2030. The basis of these studies is four visions, shown in Figure 1. These visions are very distinct from each other and they try to capture the possible realisations of random variables. It is expected that the real trajectory towards 2030 (blue arrow in Figure 1) will land somewhere within the space described by the four macroscenarios. Although a set of future macro-scenarios is intended to encompass the range of credible future out-turns, history suggests that the processes for forming such scenarios do not always succeed [9].

The macro-scenarios are used as inputs to the GARPUR system development framework. One macro-scenario is not considered either more probable or more preferred than another. Each one provides a possible picture of the future based on a macro-hypothesis. A macro-hypothesis results from judgement about possible energy policies of countries, e.g. support for energy efficiency, deployment of electric vehicles and the use of demand side management, and postulations of economic growth and use of electrical energy. A macroscenario quantifies the assumptions in a macro-hypothesis and provides capacities and types of generation, storage and demand, and also informs about the evolution of fuel and emission prices.

\section{B. Operational scenarios}

An operational scenario (or micro-scenario) is defined as a particular realisation of demand, generation and network topology. A large set of yearly time-series with, in the work reported here, one-hour temporal resolution is built for a given target year and macro-scenario. These operational scenarios capture the operational uncertainties around the actual operating states and are constrained by the maximum demand and the location and capacities of generation defined by the given macro-scenario.

Traditionally, grid development has been based on the extreme values of the demand in a country; however, due to the development of RES and interconnections between countries, there is no longer any certainty that this state is really the worst the system will face. Moreover, a worst-case scenario is not an acceptable approach if we want to quantify the expected costs related to reliability management or the likely year-round economic impact of different macro-scenarios and grid developments. For these purposes, it is necessary to generate more credible time-series that capture the full range of variability of load and RES generation patterns and their correlations. Based on the available historical data, e.g. demand, generator availability and availability of power from renewable energy sources, we generate time-series that are used to describe the real-time operation of the power system. These time series are generated with an hourly time step over a period of one year and for each area of the modelled power system. The number of time-series is increased by applying planned outages for generation and transmission system assets. A similar idea of generation of so called Monte-Carlo years is used in the system development work package of another European project, eHighway 2050 and in the Mid-Term adequacy forecast report from ENTSOE-e. (see [10]-[12] for more details).

\section{Modelling of the wholesale electricity market}

A year-long hourly time series of generation availability and demand plus relative costs of generation are used as inputs to a 'market model' to obtain a realistic commitment of thermal generation. The market model is an optimisation model that is used as a proxy for the behaviour of a wholesale electricity market. The results of the market model give complete knowledge of commitment and set-points of generation units subject to constraints on meeting given demand, minimum up and down times and ramp rates of individual generating units. This process is constrained by cross-border available transfer capacities (ATCs) but not by within-country network limits.

The market dispatch model used in this paper is a simulation tool developed by the French TSO RTE, ANTARES [13]; it performs the optimal generation dispatch of the thermal units, taking into account the grid transfer capacities between market areas.

\section{Clustering}

The commitment of thermal and hydro generation obtained in the previous step considers a time-series and respects the temporal constraints. However, for the next steps of the process, snap shot analysis is sufficient. Thus, the year-long time-series steps are converted into snapshots by ignoring the temporal links. Conversion of yearly time-series into hourly snap-shots gives rise to a very large set of operating conditions in which many operating conditions have similar characteristics. In principle, clustering can be used to identify similar cases of which only one, representative example needs to be studied in detail. In this work, two main clustering approaches were compared.

1) Clustering by k-means: This is a well known clustering method that classifies a data set through a certain number of clusters (k) fixed a priori. Clusters are grouped around centroids, i.e. data points that are nearest to the centre of its cluster. Each other data point's distance from each centroid is determined and it is associated with the cluster with the centroid to which it is nearest. As clusters change, the centroid might be changed and the association of data points with clusters is repeated until there are no further changes. Centroids can then be regarded as representative of their clusters. The feature set used for clustering in this paper consists of: demand, generation dispatch from the wholesale market model, and real power flows found by solving the load flow problem. Each of the features is in units of MW with a similar range and Euclidean distance is used as a measure of distance for the k-means algorithm. More technical details regarding the use of k-means are available in [7].

2) The old approach of clustering in Elia: Elia's former method of clustering was based on the use of some simple heuristics to select snapshots to represent system operation over a whole year. In that approach, system development 
TABLE I

BRANCH RATINGS IN THE TEST SYSTEM.

\begin{tabular}{|c|c|c|c|}
\hline & \multicolumn{3}{|c|}{$\%$ of nominal } \\
\hline Season & Lines & Transformers & Cables \\
\hline Winter & 110 & 107 & 100 \\
\hline Inter-season & 100 & 103 & 100 \\
\hline Summer & 95 & 98 & 100 \\
\hline
\end{tabular}

studies were performed using 5 clusters for the regional grids (70 kV and below) and 100 clusters for high voltage grids (150-380 kV and neighbouring transmission systems). Each of the clusters is represented by the cluster centroid, i.e. a single operating point. The 5 operating points are based on estimates of peak demand, minimum demand and average demand for summer, winter and inter-season (Spring and Autumn). The 100 operating points are obtained on the basis of demand and generation dispatch from the wholesale market model and flows on transmission assets found by solving the load flow problem. The method for obtaining the 100 operating points is described in [14].

Following successful completion of the first phase of the system development framework of GARPUR [7], Elia has now started using the k-means approach to clustering for system development studies. In this paper, the old approach of clustering in Elia is used for comparison with the k-means approach.

\section{E. Modelling of transmission system operator actions}

The operability of the centroids of each cluster, i.e. possible future operating states, is assessed using an optimisation model that assumes the form of a security-constrained optimal power flow (SCOPF) [15], [16]. The optimisation models the behaviour of a transmission system operator and the objective function is to minimise the cost of re-dispatch from a market proposed solution. In order to ensure that an OPF solution is found, the permissible actions include curtailment of RES and some moderate post-contingency overloading of transmission assets relative to their continuous ratings. The seasonal ratings of transmission lines, transformers and cables are given in Table I. The results of the OPF are then used to assess the need, or otherwise, for investment in new network facilities. The mathematical details regarding the objective function and constraint set of the optimisation problem are available in [7].

We note that the costs of balancing a system using generation regulation (accepting bids and offers) cannot be forecasted for a very long-term horizon. For the testing reported here, a relative cost order was assigned to the balancing actions that reflects the actions available to the system operator in Belgium and the way the current regulatory environment determines preferences. The relative costs are used to guide the optimisation in a similar direction to that which would be used by the system operator and are shown in Table II.
TABLE II

PSEUDO-COSTS USED IN THE ASSESSMENT MODEL.

\begin{tabular}{|l|c|}
\hline Balancing action & Pseudo-cost (Euros/MWh) \\
\hline Load-shedding & 7000 \\
\hline PV curtailment & 3000 \\
\hline Wind curtailment & 1000 \\
\hline Conventional generation re-dispatch & 100 \\
\hline Overloading of unsupervised assets & 0.1 \\
\hline
\end{tabular}

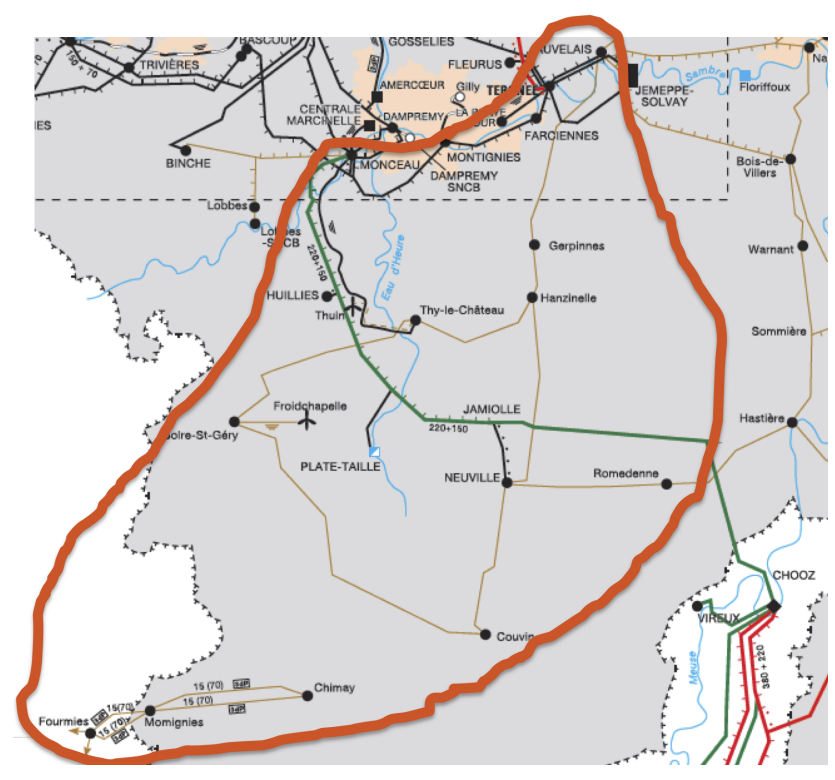

Figure 2. South-Western part of the High-voltage Belgian transmission network.

\section{Test network: A Part of The Belgian TRANSMISSION SYSTEM}

A part of the Belgian transmission system, located in the South-West of Belgium bordering France, is considered in order to demonstrate the GARPUR system development framework. The chosen network consists of a rural 70-150 kV transmission grid (Figure 2). For the year 2030 and under a macro-scenario of 'slow progression' this part of the Belgian network includes generation capacity made up of approximately $30 \%$ wind, $11 \%$ pumped storage and $5 \%$ photo-voltaic $(\mathrm{PV})$, respectively (Table III). Such a diverse mix of generation makes this part of the Belgian network an interesting case study for system development.

TABLE III

GENERATION MIX IN THE TEST SYSTEM FOR YEAR 2030.

\begin{tabular}{|c|c|c|}
\hline Generation type & Number of generators & Generation capacity(\%) \\
\hline Gas & 22 & 43.8 \\
\hline Wind & 20 & 30.6 \\
\hline Pump & 4 & 10.7 \\
\hline Biogas & 9 & 10.0 \\
\hline PV & 95 & 4.7 \\
\hline Hydro & 3 & 0.2 \\
\hline
\end{tabular}


This grid contains eight $70 \mathrm{kV}$ substations, $110 \mathrm{~km}$ of $70 \mathrm{kV}$ lines, $34 \mathrm{~km}$ of $150 \mathrm{kV}$ transmission lines and $21 \mathrm{~km}$ of $150 \mathrm{kV}$ cables. It is fed by two $150 / 70 \mathrm{kV}$ transformers located at Neuville and Thy-le-Château and are supported by two interzonal $70 \mathrm{kV}$ transmission lines. Both 150/70 kV transformers operate in parallel on same $150 \mathrm{kV}$ substation (one via a cable and the other via a line). The $70 \mathrm{kV}$ substations supply relatively low electricity demand (peak of each substation between 5 and 15 MVA) and are linked with long lines that are reaching the end of their life. Most of the substations are only fed by two lines, which means that a loss of load is expected if one transmission line is on planned outage and an unplanned outage occurs at the second. The risk of such loss of load is accepted by the TSO because the impact of loss of load at a single $70 \mathrm{kV}$ substation is relatively small.

In addition to a need for investment in this area to replace ageing assets, the area is very conducive to the development of wind power generation. Currently, $60 \mathrm{MVA}$ of wind generation is connected (one wind farm directly on $70 \mathrm{kV}$ grid, the rest at lower voltage levels) and 130 MVA have reserved capacity. In addition, the total potential for wind generation is estimated at 400 MVA. Furthermore, of the 130 MVA currently reserved, $45 \mathrm{MW}$ have non-firm access to the $70 \mathrm{kV}$ grid. In the event of unplanned outages, production at these wind farms can be curtailed at no cost to the TSO.

\section{GenERATION AND ASSESSMENT OF OPERATIONAL SCENARIOS FOR THE BELGIAN NETWORK}

The complete Belgian transmission network consists of approximately 11,000 nodes. The DigSilent PowerFactory [17] network reduction toolbox was used to obtain a reduced network that models the area highlighted in Figure 2 explicitly but collapses the external network to a small number of equivalent nodes, branches and injections. The reduced network consists of 132 nodes and 261 branches and transformers.

\section{A. Generation of operational scenarios}

A target year of 2030 under the 'slow progression' macroscenario from the ENTSO-E vision [8] was adopted to demonstrate the use of the proposed framework. However, the same approach can be used for any other macro-scenario and year.

Demand data for 2030 were constructed based on the historical records of demand. Elia has such records for all medium voltage substations with a temporal resolution of 15 minutes. Although quite significant changes to patterns of demand can be envisaged by 2030, these were not features of the 'slow progression' macro-scenario. Thus, in this study, it was assumed that demand will be similar in 2030, scaled by the peak demand set in the macro-scenario. The RES generation profiles for PV and wind were obtained using ENTSO-e's Pan-European Climate Data Base [10]. Note that even with a single year of considered operation, 8736 different realisation of demand and RES generation are considered.

Once time-series of demand and generation from RES have been determined, they are passed to the market dispatch tool ANTARES, which determines a time-series of commitment and set-points of thermal generation. This gives 8736 hourly snapshots (364 days) for each 'Monte Carlo year'.

\section{B. Assessment of snapshots for operability of the system}

In this section, we assess 8736 snapshots and analyse the performance of the two approaches to clustering. A (N-1) contingency list is used in the SCOPF model. 'Supervised' assets are the overhead lines, underground cables and transformers that are monitored by Elia and whose contingencies are considered in operational time frames. Thermal loading limits on supervised assets are applied as hard constraints in the SCOPF and their outages form the contingency list. Contingencies for 'unsupervised' assets are not considered in the analysis. However, Elia's experience suggests that binding contingencies arise only for supervised assets. Instead of applying hard constraints to unsupervised assets, a low cost is set for overloading them. This is in order to give some degree of freedom to the SCOPF so that solutions can be found but primarily in order to find out which unsupervised assets are overloaded so that the need for reinforcement of such transmission corridors can be identified. After solving the SCOPF, one of the following three labels is assigned to each snapshot:

- Safe: Pre-contingency and post-contingency flows are within operating limits and all demand is met.

- Unsafe in (N-1): Safe in the pre-contingency state but there are one or more post-contingency violations of operating limits.

- Unsafe in $\mathbf{N}$ and (N-1): Violation of operational limits in pre- and post-contingency states.

The assessment results show that no load shedding (pre- or post-contingency) occurs in any of the snapshots. In $3.7 \%$ of the snapshots wind was curtailed in pre-contingency operation and in $15.6 \%$ of snapshots wind was curtailed during postcontingency operation. The particular contingencies that lead to the curtailment of wind were identified. Cases with high outputs from certain wind farms that led to overloading of network assets were also identified. (In all these cases, the overloaded assets were specific transformers).

Figure 3 shows the assessment results of all the 8736 snapshots. It may be observed that $86 \%$ of the snapshots are Safe, $10 \%$ of the snapshots are Unsafe in (N-1) and $4 \%$ are Unsafe in $\mathrm{N}$ and (N-1) states. It is important to note that, as shown in Figure 3, the Unsafe operating states do not occur at maximum demand. However, peak wind cases result in overloading of network branches and that is where most of the problematic cases exist.

Table IV presents the results of clustering of 8736 snapshots and the assessment of system operability based on analysis only of the centroids of each cluster. It can be seen that Elia's former approach of using 5 operating states is a very poor representation of a full year; it under-estimates the cost of generation re-dispatch by $98.8 \%$ and misses all the cases that are Unsafe in $\mathrm{N}$ and $\mathrm{N}-1$. The k-means clustering with 100 clusters performs better in capturing safe operating states with a small $0.9 \%$ over-estimation. For k-means clustering, the error 
TABLE IV

COMPARISON OF ASSESSMENT RESULTS USING DIFFERENT APPROACHES TO CLUSTERING

\begin{tabular}{llccccc}
\hline & & All data & \multicolumn{2}{c}{ k-means } & \multicolumn{2}{c}{ Elia's old approach } \\
\cline { 3 - 7 } & & & 100 clusters & 870 clusters & 5 clusters & 100 clusters \\
\hline \multirow{2}{*}{ Safe } & [hours] & 7499 & 7567 & 7528 & 6984 & 7735 \\
& Relative error & - & $0.9 \%$ & $0.4 \%$ & $-6.9 \%$ & $3.1 \%$ \\
\hline \multirow{2}{*}{ Unsafe in N-1 } & [hours] & 914 & 949 & 866 & 1752 & 777 \\
& Relative error & - & $3.8 \%$ & $-5.3 \%$ & $91.7 \%$ & $-15.0 \%$ \\
\hline \multirow{2}{*}{ Unsafe in N and N-1 } & [hours] & 323 & 221 & 342 & 0 & 224 \\
& Relative error & - & $-31.6 \%$ & $-5.9 \%$ & $-100.0 \%$ & $30.7 \%$ \\
\hline Operating cost & Relative error & - & $-8.9 \%$ & $-4.1 \%$ & $-98.8 \%$ & $15.2 \%$ \\
\hline
\end{tabular}

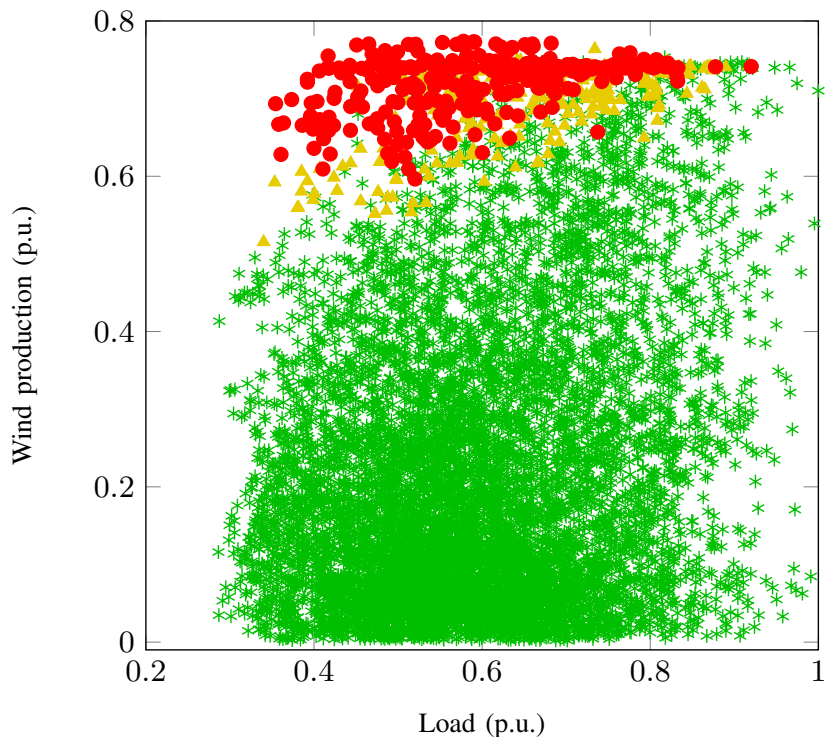

Figure 3. Secure and insecure operating states for 8736 (one-year) snapshots. Secure operating states are those where no breaches of operational limits occur in pre- or post-contingency operation, i.e. are Safe (green stars). Amber triangles represent cases that are Unsafe in $(\mathrm{N}-1)$ and red circles represent those that are Unsafe in $\mathrm{N}$ and $(\mathrm{N}-1)$.

decreases when the number of clusters is increased to 870 . The value of 870 is used because it represents approximately $10 \%$ of the total number of snapshots. However, this more than 8-fold increase in the number of clusters results in only a $50 \%$ reduction in the classification error. This small decrease is because of the inability of the clustering algorithm as formulated to identify high impact low probability (HILP) cases.

Figure 4 shows a comparison between the clustering methods. The vertical axis of the figures shows the normalised costs of operation, i.e. the cost of generation re-dispatch plus any incurred pseudo-costs of overloading of unsupervised network branches and load shedding. It can be seen that none of the clustering approaches succeeds in capturing the few percent of snapshots that have the highest costs.

Figure 5 shows the comparison between Elia's former approach and k-means clustering with $k=100$. For the most

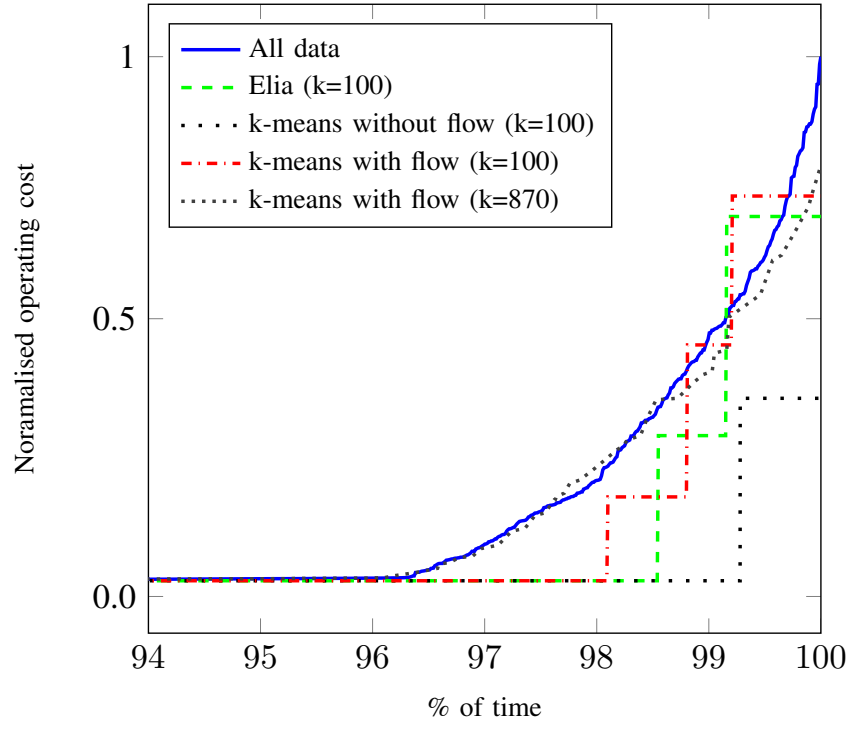

Figure 4. Cumulative distributions of operating cost (OPEX) as estimated by analysis of centroids of clusters identified using different methods

part, the methods have comparable representation of time in each centroid i.e. similar numbers of cases in each cluster exemplified by corresponding centroids. Figure 5(b) shows the standard deviation in k-means method is much smaller as compared to Elia's approach. This suggests that the kmeans method performs better at suggesting centroids that have relatively good representations of operating cost within each cluster.

\section{Assessment of INVESTMENT CANDIDATES}

A critical centroid is defined as an operating case that is Unsafe in either $\mathrm{N}$ or $\mathrm{N}-1$ and should be examined further by the system planner in order to assess whether reinforcement might be needed. The other cases in the same cluster as a critical one are also labelled as critical. Table $\mathrm{V}$ shows the number of critical centroids highlighted by each method for examination by the planner. The table also shows the number of operating states revealed by analysis of each and every one to be Unsafe but which are not captured by the critical centroids. For k-means with 870 clusters, 105 centroids are 


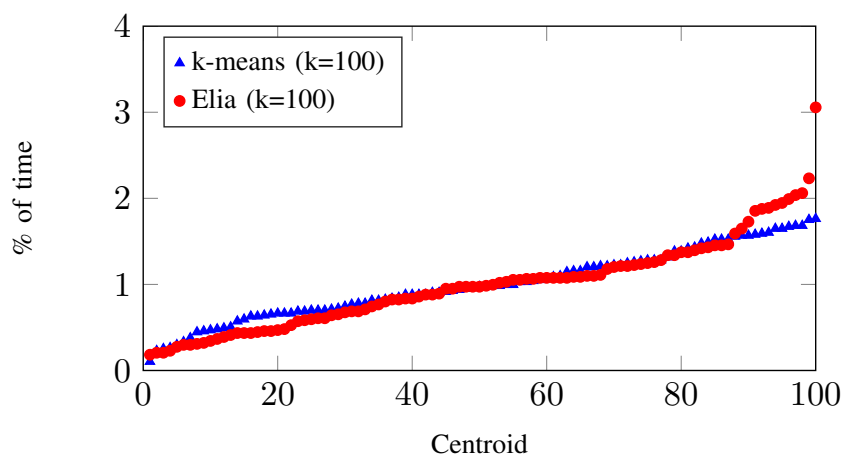

(a) Representation of time corresponding to each centroid.

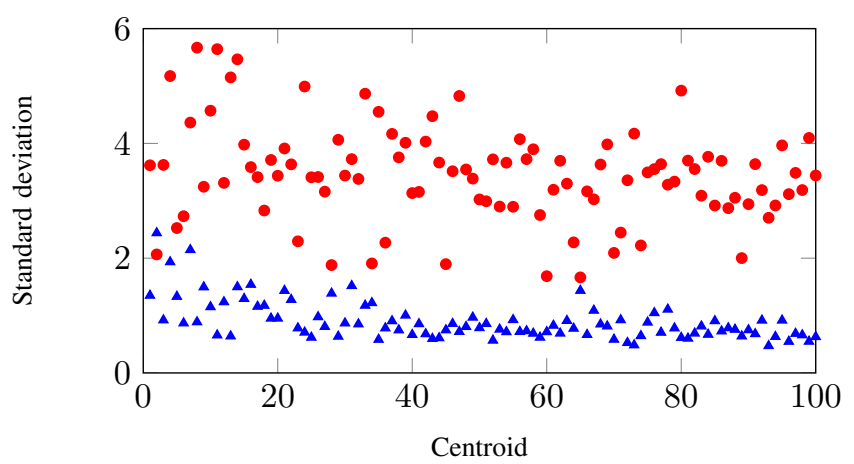

(b) Standard deviation in each centroid.

Figure 5. Comparison between k-means and Elia's old approach of clustering.

TABLE V

NUMBER OF CRITICAL CENTROIDS.

\begin{tabular}{llll}
\hline & \multicolumn{2}{c}{ k-means } & Elia \\
\cline { 2 - 4 } Number of clusters & 100 & 870 & 100 \\
\hline Number of critical centroids & 15 & 105 & 7 \\
\hline $\begin{array}{l}\text { Unsafe operating states } \\
\text { missing from critical clusters }\end{array}$ & 104 & 118 & 261 \\
\hline
\end{tabular}

identified as critical. This is a large number of operating states to be studied in detail, even for an expert. Practical experience in Elia suggests that 10 is a practical number for a system planner to investigate for proposing future investments in the grid. For that reason, the 10 most expensive centroids are recommended for further investigation.

Careful study of the chosen centroids suggests that the operating cost can be reduced by reinforcement of the part of the network where wind is most often curtailed. The assessment results on these centroids identify the transmission corridors that are loaded up to their capacity and this indicates that increasing transmission capacities in those corridors would reduce the curtailment of wind generation. We note that if no grid reinforcement is done and the transmission assets that are coming to end of their life are simply retired rather than replaced, then the operating cost for the 2030 macroscenario is 21 times more than with the existing, 2017 network infrastructure.

Two investment options were identified for this grid. These both propose to mesh the two $150 \mathrm{kV}$ peninsulas. However, the first one, Option-A, splits the meshed part of the $70 \mathrm{kV}$ and supports the rest of $70 \mathrm{kV}$ grid via two $150 / 70 \mathrm{kV}$ transformers. In this option, some substations are moved from $70 \mathrm{kV}$ to $150 \mathrm{kV}$ voltage level. The second, Option$\mathrm{B}$, consists of keeping the $70 \mathrm{kV}$ grid meshed, retaining the $70 \mathrm{kV}$ substations and supporting it via 3 additional 150/70 kV transformers of higher capacity than the existing ones.

The two network development options described above are compared with that of the existing network infrastructure (Option-E) and a 'do-nothing' option (Option-0) that retires end-of-life assets without replacement and, otherwise, retains the existing network infrastructure (Table VI). Their effects on system operability are summarised in Figure 6. We note that in Option-0 the number of Unsafe operating conditions in $\mathrm{N}$ and $(\mathrm{N}-1)$ increases by 1.8 times (Figure 6(a)) and the operating cost increases by 5 times. Figure 6(b) is normalised by the cost of option-E. All 8736 assessed operating states are Safe for Option-A, and the operating cost is zero. Some operating states are Unsafe in $(\mathrm{N}-1)$ for Option-B, and the operating cost is non-zero because a control action is required by the system operator to correct the solution. However the operating cost is small compared to that with the existing topology.

In principle, investment decisions are finally made on the basis of maximised total social welfare where social welfare is the sum of 'producer surplus' (the surplus of generators' revenues, determined by the market price, over their costs) and 'consumer surplus' (the total by which the amount paid by consumers for energy is less than what they were willing to pay) [18]. The amount paid by consumers will finally include the cost of the network's infrastructure. In practice, because of the difficulty of knowing each generator's costs and consumers' willingness to pay, TSOs make decisions based on miminisation of the sum of capital costs (for network assets) and operating costs (predominantly for dispatch or re-dispatch of generation or, where available, flexible demand) over the medium to long-term [19]. The capital costs of reinforcements can only be known with confidence once detailed specifications have been formed and a tendering exercise carried out for which the results are often commercially confidential. The first aim of investment planning is to identify network reinforcement options and the dates by which they might need to be exercised. Depending on the nature of the reinforcement and how long it might take to deliver, a re-evaluation is then carried out a minimum of 2 years before the required date of commissioning. At that point a reasonable estimate of absolute not just relative operating costs should be possible allowing a cost-benefit analysis comparing the benefits of reinforcement with its cost. In the case study reported here, it is concluded that Option-B could only be optimal if its capital cost is at least $10 \%$ less than that of Option-A.

\section{DisCUSSION AND CONCLUSIONS}

In this paper, the application of the system development framework proposed in the European research project 
TABLE VI

TRANSMISSION INVESTMENT OPTIONS.

\begin{tabular}{ll}
\hline Option & Context \\
\hline $\mathrm{E}$ & $\begin{array}{l}\text { Like-for-like replacement of aged assets and } \\
\text { retention of the existing topology }\end{array}$ \\
0 & $\begin{array}{l}\text { Do nothing. Remove the assets coming to end of } \\
\text { their life }\end{array}$ \\
$\mathrm{A}$ & $\begin{array}{l}\text { Reinforcement by mainly investing in the } 70 \mathrm{kV} \text { grid } \\
\mathrm{B}\end{array}$ \\
\hline
\end{tabular}

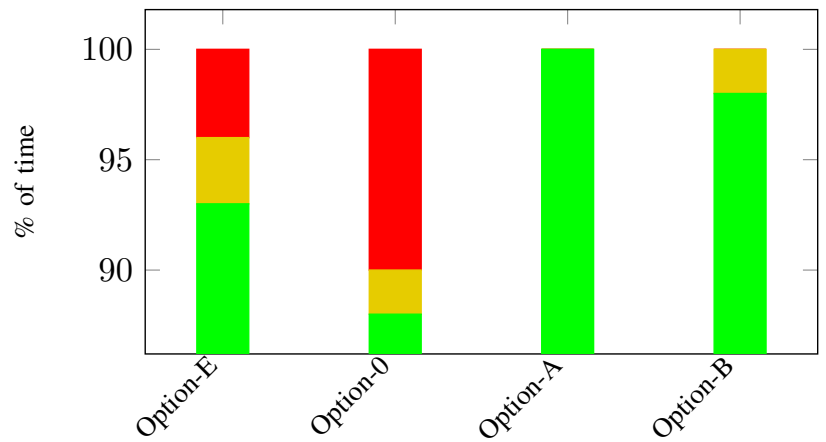

(a) Percentage of Safe (green) and Unsafe (amber and red) operating conditions for each investment option.

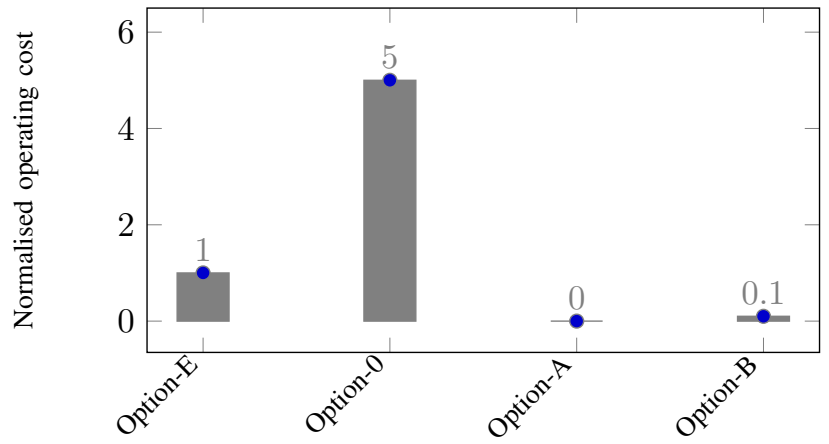

(b) Operating cost for each investment option. The vertical axis of the figure is normalised by the cost of Option-E.

Figure 6. Results of implementation of investment options.

GARPUR has been demonstrated. It is shown that the proposed framework can be implemented on a real transmission network, in this case the one in Belgium for a particular future macro-scenario. The importance of considering wide variety of possible scenarios and contexts has been highlighted. These include both macro-scenarios and operational scenarios. Conventional current practice in system development planning does not, in general, cover many different operating conditions and, as a consequence, risks either over-investment (because the few conditions that are assessed are onerous and not representative of year-around risks and trade-offs) or under-investment (because some critical conditions have been missed). The approach described in this paper synthesises a large number of credible operating states covering a full year of operation of under a particular macro-scenario but manages the workload on system development engineers (a) by assessing the operability of the macro-scenario for a given network infrastructure for a limited number of representative cases, identified by clustering and (b) using a security-constrained OPF to model how an operating state would be managed by the system operator. However, testing of the approach reveals some important directions for further work, discussed below.

\section{A. Limitations of clustering approaches}

The k-means approach and an approach previously developed by the Belgian TSO, Elia, have been compared in this paper. However, both have limitations.

1) Both approaches require the number of clusters to be set in advance, regardless of the trade off between how well they might succeed in covering the problem space and the practicality of more detailed assessment of a large number of cases.

2) For a given number of clusters, the approach used might succeed in identifying clusters with consistently moderate variability within each cluster (as shown by the standard deviations for k-means in Figure 4) and in identifying centroids that are representative of the clusters. However, even with a large number of clusters, these might fail to adequately capture the relatively few credible individual operating states that would present very significant problems for system operation, i.e. either high cost of re-dispatch of generation or high risk of failing to meet demand. Moreover, simple metrics of adverse impacts may fail to capture the disproportionately large cost of large disturbances [20].

\section{B. Maintenance}

A very important dimension of a TSO's job is the management of the network's assets and the facilitation of outages to allow them and networks users' assets to be maintained so that their reliability is acceptable in the medium to long term. A system development planner must ensure that the system can be operated in future not only under initially intact system conditions but also when maintenance or construction work (to implement a reinforcement or replace an aged asset) is being carried out. This might be assessed either in respect of the operability of the future system given a credible schedule of planned outages or by ensuring, for each area of the system, that there is a sufficient window of time in which at least one planned outage might be taken while not impacting excessively on operability. The GARPUR project has performed some initial investigation into these issues and how an optimal outage schedule might be formed. (See [7], [21]). However, more work is required to ensure that the approach used is both informative and practical for system developing planning.

\section{REFERENCES}

[1] GARPUR Consortium, "Functional analysis of system development process," Deliverable 4.1, 7th framework programme, EU Commission grant agreement 608570, Tech. Rep., March 2015. [Online]. Available: http://www.garpur-project.eu/deliverables

[2] European Commission, "The unbundling regime: Interpretative note on directive 2009/72/ec concerning common rules for internal market," Tech. Rep., 2010. 
[3] European Network of Transmission System Operators for Electricty (ENTSO-E), "Overview of internal electricty marketrelated project work," Tech. Rep., 2014. [Online]. Available: https://www.entsoe.eu/Documents/Events/2014/141013_ENTSOE_Update-on-IEM-related 20project 20work_final.pdf

[4] European Parliament, "Directive 2009/28/ec of the european parliament and of the council of 23 april 2009 on the promotion of the use of energy from renewable sources," Tech. Rep., 2009. [Online]. Available: http://eur-lex.europa.eu/eli/dir/2009/28/oj

[5] European Energy Regulators White Paper 1, "Renewables in the wholesale market," Tech. Rep., 2017. [Online]. Available: https://www.ceer.eu/en/white-papers

[6] GARPUR Consortium, "Current practices, drivers and barriers for new reliability standards," 7th framework programme, EU Commission grant agreement 608570, Tech. Rep., May 2014. [Online]. Available: http://www.garpur-project.eu/deliverables

[7] - "Upgrading of the decision-making process for system devlopment," Deliverable 4.2, 7th framework programme, EU Commission grant agreement 608570, Tech. Rep., January 2017. [Online]. Available: http://www.garpur-project.eu/deliverables

[8] European Network of Transmission System Operators for Electricty (ENTSO-E), "Ten-year network development plan 2016," Tech. Rep., 2016. [Online]. Available: http://tyndp.entsoe.eu/

[9] E. Trutnevyte, W. McDowall, J. Tomei, and I. Keppo, "Energy scenario choices: Insights from a retrospective review of UK energy futures," Renewable and Sustainable Energy Reviews, vol. 55, no. Supplement C, pp. 326 - 337, 2016. [Online]. Available: http://www.sciencedirect.com/science/article/pii/S1364032115011466

[10] European Network of Transmission System Operators for Electricty (ENTSO-E), "Mid-term adequacy forecast 2017 edition," Tech. Rep., 2017. [Online]. Available: https://consultations.entsoe.eu/systemdevelopment/mid-term-adequacy-forecast-2017/supporting_documents/

[11] K. Adam, M. Mller-Mienack, M. Paun, G. Sanchis, and K. Strunz, "eHIGHWAY 2050; The ENTSO-E facilitated study programme towards a modular development plan on pan-European electricity highways system 2050," in 2012 IEEE Power and Energy Society General Meeting, July 2012, pp. 1-6.

[12] e-Highway 2050 Consortium, "Detailed enhanced methodology for long-term grid planning," Deliverable 8.6a, 7th framework programme, EU Commission grant agreement 308908, Tech. Rep., 2012. [Online]. Available: http://www.e-highway2050.eu/results/

[13] M. Doquet, C. Fourment, and J. M. Roudergues, "Generation and transmission adequacy of large interconnected power systems: A contribution to the renewal of Monte-Carlo approaches," in 2011 IEEE Trondheim PowerTech, June 2011, pp. 1-6.

[14] P. V. Roy, "A practical implementation of representative plan case selection for grid studies, as used in TYNDP studies for ENTSO-E," in CIGRE session, C1-319, 2016.

[15] O. Alsac and B. Stott, "Optimal load flow with steady-state security," IEEE Transactions on Power Apparatus and Systems, vol. PAS-93, no. 3, pp. 745-751, May 1974.

[16] A. Monticelli, M. V. F. Pereira, and S. Granville, "Security-constrained optimal power flow with post-contingency corrective rescheduling," IEEE Transactions on Power Systems, vol. 2, no. 1, pp. 175-180, Feb 1987.

[17] DIgSILENT GmbH., "DIgSILENT PowerFactory-user manual," Tech. Rep., 2016.

[18] GARPUR Consortium, "Recommendations for implementing the socio-economic impact assessment methodology," Deliverable 3.2, 7th framework programme, EU Commission grant agreement 608570 Tech. Rep., March 2015. [Online]. Available: http://www.garpurproject.eu/deliverables

[19] CIGRE Working Group C1.24, "Tools for economically optimal transmission development plans," Technical Brochure 572, CIGRE, Tech. Rep., 2014.

[20] W. A. Bukhsh, K. R. W. Bell, and T. Bedford, "Risk and reliability assessment of future power system," in In European Safety and Reliability Conference, Glasgow, 2016.

[21] GARPUR Consortium, "Pathways for mid-term and long-term asset management," Deliverable 5.2, 7th framework programme, EU Commission grant agreement 608570, Tech. Rep., March 2015. [Online]. Available: http://www.garpur-project.eu/deliverables 